\title{
¿Son las cenizas volcánicas un riesgo para la salud respiratoria? Revisión a propósito de la erupción del cordón del Caulle en junio de 2011
}

\author{
EDGARDO GROB B.*,****, MANUEL OYARZÚN G.**,****, ISABEL CAVIERES C.***, \\ PABLO ZARGES T.*** y GABRIEL BUSTAMANTE M.***
}

\begin{abstract}
Are volcanic ashes a risk for the respiratory health? A review apropos of volcanic eruption of cord of Caulle, Chile in June 2011
\end{abstract}

Chile is a volcanic country with over 500 active volcanoes. Mounts Llaima and Villarrica are among the four most active volcanoes in South America. Activation or reactivation of a volcano is an unpredictable phenomenon and its impact can be catastrophic. Volcanic eruptions can trigger a variety of hazardous events, such as explosions, pyroclastic flows, mudflows, acid rain, climatic effects, among others. The impact of a volcanic eruption on human health depends on the type of eruption at which inhabitants have been exposed, the duration of this exposure and the previous health conditions of the exposed people. Some of these effects on human health are well known, such as burns, trauma, and respiratory disorders. In acute as well as in long term exposure, some of the respiratory health effects are still a matter of controversy and research on the mechanisms involved. Among the main adverse effects described in the respiratory tract are bronchoconstriction, amplification of the inflammatory response, complement disorders, silicosis and carcinogenesis. Considering the recent volcano eruptions of mount Chaitén (2008) and the cord of Caulle (2011) located in the south of Chile, we reviewed the deleterious effects of volcanic eruptions on human health and specifically on the respiratory system. Throughout this review we enclosed a number of recommendations, hopefully they will be useful in facing new volcanic eruptions in our country and everywhere.

Key words: Volcanic eruptions, volcanic ashes, human health, lung disease.

\section{Resumen}

Chile es un país volcánico con más de 500 volcanes activos. Los volcanes Llaima y Villarica están entre los cuatro más activos de Sudamérica. La activación o reactivación de un volcán es un fenómeno impredecible y su impacto puede llegar a ser catastrófico. Las erupciones volcánicas contemplan diversas manifestaciones, tales como explosiones, flujos piroclásticos, flujos de lodo, lluvia ácida, efectos climatológicos, entre otros. El impacto sobre la salud humana depende del tipo de manifestación a la que haya sido expuesta una determinada población, la duración de esta y las condiciones de salud previas a la exposición. Algunos de estos efectos son ampliamente conocidos, como quemaduras, traumatismos y alteraciones del sistema respiratorio. Siendo algunas de estas últimas aun motivo de controversia e investigación, tanto en sus efectos agudos y a largo plazo como en sus mecanismos. Dentro de las principales alteraciones descritas en el sistema respiratorio se encuentran la broncoconstricción, amplificación de la respuesta inflamatoria, alteraciones del complemento, silicosis y carcinogénesis. Considerando las recientes erupciones volcánicas registradas en el sur de Chile: Volcán Chaitén (2008)

* Profesor Adjunto, Instituto de Medicina y Director Campo Clínico Osorno. Facultad de Medicina, Universidad Austral de Chile. Osorno XIV Región.

** Profesor Titular, Director Programa de Fisiopatología, Instituto de Ciencias Biomédicas, Facultad de Medicina, Universidad de Chile, Santiago.

*** Alumnos de Medicina, Universidad Austral de Chile. Osorno XIV Región.

**** Comisión de Contaminación Ambiental, Sociedad Chilena de Enfermedades Respiratorias. 
y el cordón del Caulle (2011), hemos realizado una revisión de los efectos deletéreos de éstas sobre la salud humana y especificamente sobre el sistema respiratorio. Esperamos que esta revisión y la serie de recomendaciones incluidas en ella, sean de utilidad ante una nueva erupción volcánica en Chile y en cualquier otro país.

Palabras clave: Erupción volcánica, cenizas volcánicas, salud humana, enfermedad pulmonar.

\section{Introducción}

Las grandes erupciones volcánicas que se han registrado en nuestro país en la localidad de Chaitén $(2 / 5 / 2008)$ situado en Chiloé continental y más recientemente $(4 / 6 / 2011)$ en el cordón del Caulle (1.430 msnm) ubicado en la Región de los Lagos, nos han motivado a realizar una revisión bibliográfica sobre los efectos de las erupciones volcánicas en la salud de la población expuesta y más específicamente en el sistema respiratorio y las medidas que se pueden tomar para protegernos de esta catástrofe natural.

En esta revisión fueron consultadas las bases de datos Medline y Lilacs. A través de los sistemas PubMed y Bireme, se llevó a cabo una revisión de la literatura científica entre 1980 y 2012. Las palabras clave usadas en la búsqueda bibliográfica fueron: volcanic eruption y volcanic ashes, asociadas a los términos health, human health, lung diseases, respiratory effects o prolonged exposure.

A lo largo de la cordillera de los Andes y sólo en el territorio chileno, existen más de tres mil volcanes. A pesar que muchos de ellos han estado inactivos hasta ahora, actualmente existen alrededor de quinientos considerados activos. De estos, existen sesenta con registro eruptivo histórico dentro de los últimos 450 años, provocando daños en las personas, bienes materiales y ambiente ${ }^{1}$. Los volcanes Villarrica y Llaima están entre los cuatro volcanes más activos de Sudamérica (Figura 1).

La actividad volcánica es uno de los fenómenos naturales más temidos y respetados. $\mathrm{Su}$ acción transformadora de la geomorfología del paisaje y sus efectos en la salud pública, hacen de las erupciones, un proceso complejo para las poblaciones afectadas por sus efectos.

El comportamiento de los volcanes no obedece a ninguna ley de periodicidad. Las erupciones aun no se pueden prevenir, aunque la detección de sismos o fumarolas pueden anunciarlas. La violencia eruptiva dependerá de la temperatura, acidez y contenido gaseoso de las lavas.

El contenido volcánico se encuentra a altas presiones producto de la elevación de la temperatura del magma, por lo que cuando la presión vence la resistencia del reservorio rocoso que lo contiene, escapa explosivamente. Las consecuencias de esta erupción son entre otras el derretimiento de hielos, derrumbes, aluviones, nubes volcánicas y la diseminación atmosférica de cenizas y gases (Figura 2).

Los principales riesgos producidos por una erupción volcánica son los siguientes:

Flujos de Lava: Constituidos por rocas licuadas emitidas por la erupción. Puede alcanzar velocidades de hasta $70 \mathrm{~km} / \mathrm{h}$ y su nivel de destrucción radica en su alta temperatura, caudal y velocidad de desplazamiento.

Explosiones: Fragmentos de roca y lava conducidos por gases disueltos en lo profundo del magma. Pueden dispersarse a kilómetros de distancia.

Flujos piroclásticos: Masas densas de gas y lava generadas a altas temperaturas y fluyen hacia abajo por las laderas del volcán. Pueden flotar o arrastrarse dependiendo de las proporciones de sus componentes.

Flujos de lodo: Combinaciones de componentes emanados del volcán (lava, gases, ceniza) con elementos ambientales (nieve, tierra, lluvia), pudiendo generar avalanchas.

Lluvia ácida: Lluvia que cae a través de una nube que libera gas, disolviendo componentes de la nube formando ácido sulfúrico y clorhídrico.

Maremotos o "Tsunamis" y marejadas: La erupciones de volcanes submarinos o de volcanes cercanos a la costa pueden generar olas de más de cinco metros producidas por sacudidas subterráneas.

Efectos climatológicos: La erupción puede producir enfriamiento o calentamiento de la superficie terrestre. El enfriamiento se debe al polvo volcánico en suspensión que refleja la luz solar desde la estratósfera, actuando como un escudo.

Impacto psicológico: La población de la zona de la erupción puede presentar alteraciones psicosomáticas como fatiga, náuseas o cefalea, alteraciones del comportamiento como trastornos del sueño y/o apetito y alteraciones del ánimo como depresión, ansiedad o manifestaciones de estrés postraumático.

Daños socioeconómicos: Accidentes de tránsito por disminución de la visibilidad, suspensión o desviación del tránsito aéreo, daño en campos y cultivos, contaminación de aguas y desabastecimiento por aislamiento (Figura 3). 


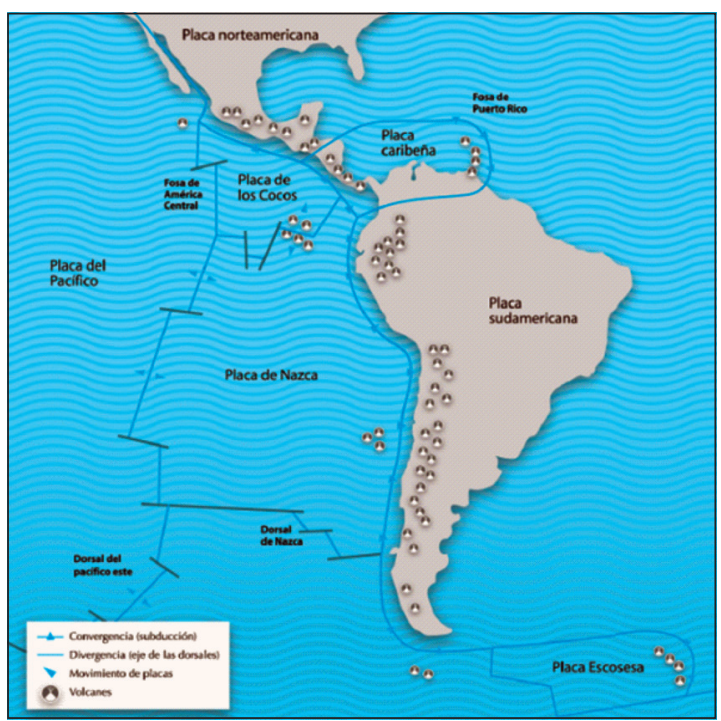

Figura 1. Distribución de los volcanes en América Central y Sud América. Extractada de la Guía de preparativos de salud frente a erupciones volcánicas, $\mathrm{OMS}^{2}$.

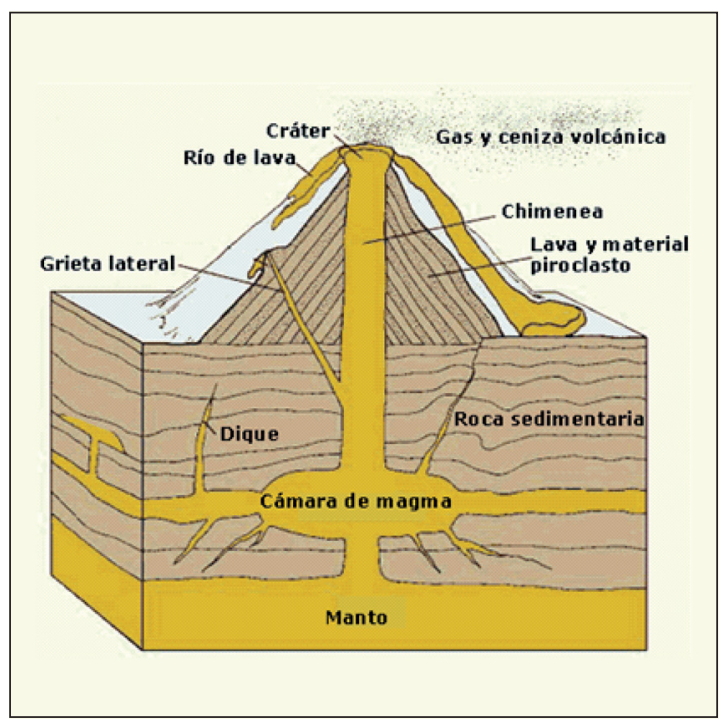

Figura 2. Estructura de un volcán en actividad. Extractada de la Guía de preparativos de salud frente a erupciones volcánicas, $\mathrm{OMS}^{2}$.

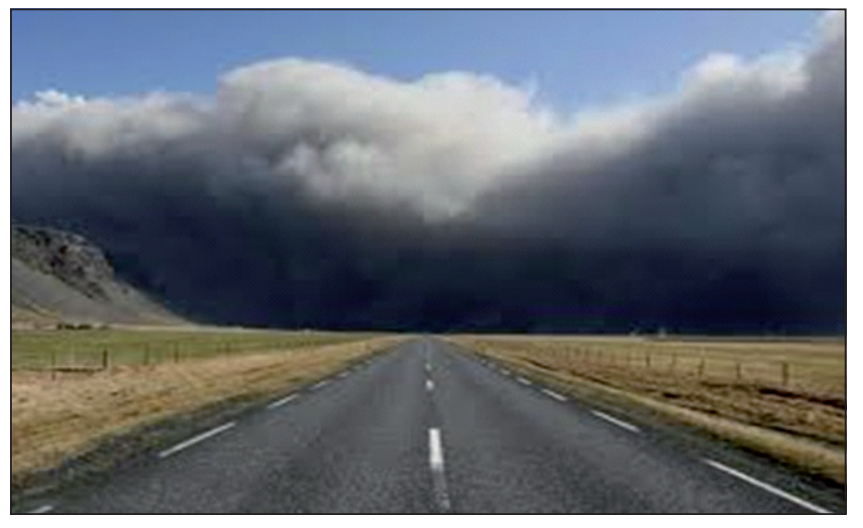

Figura 3. Camino Villa Angostura-Bariloche, Argentina. Foto de turistas al enfrentar nube de esmog volcánico del Caulle, localidad situada en línea recta cerca de $100 \mathrm{~km}$ al sur de la erupción, captada en agosto de 2011.
Desde el punto de vista epidemiológico, un evento volcánico, pone en riesgo a la población involucrada, por una serie de factores que incrementan significativamente la morbilidad. Una de las actividades sanitarias importantes frente a una amenaza de erupción volcánica es la identificación del tipo de enfermedades que pueden emerger y sus factores potenciadores. La información previa de las poblaciones en riesgo junto con la evaluación de daños, debiera servir para identificar factores de riesgo.

Entre los factores de riesgo para el aumento de enfermedades se han señalado los siguientes:

- Aumento repentino de la densidad poblacional de un sitio por desplazamiento de la población a lugares con menos riesgo.

- Contaminación en zonas de abastecimiento, agua potable, electricidad, etc.
- Cambios ambientales que pueden favorecer el desarrollo de vectores o patologías preexistentes. Un ejemplo es la exacerbación de la sintomatología respiratoria pre-existente por inhalación de ceniza volcánica.

- Interrupción de los controles de salud debido al aislamiento geográfico.

- Desplazamiento de animales, favoreciendo las zoonosis.

- Manifestaciones psicosociales por pérdidas y estrés postraumático entre otras.

Los efectos somáticos en la salud de la población por una erupción volcánica están determinados por las emisiones de cenizas y gases. La ceniza volcánica es un material de grano fino (menor de $2 \mathrm{~mm}$ ), compuesto por roca pulverizada debido a la altísima presión del vapor dentro 


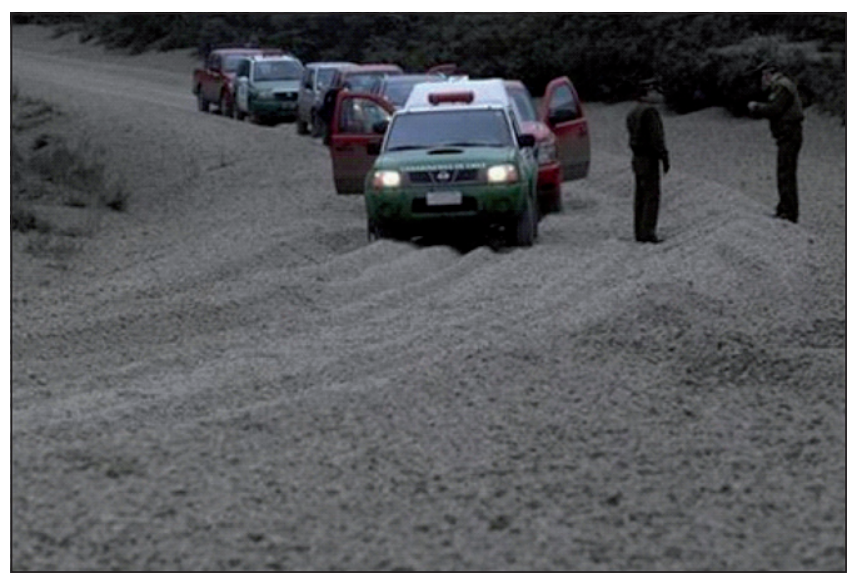

Figura 4. Acumulación de ceniza volcánica del cordón del Caulle en el paso fronterizo entre Argentina y Chile, Cardenal Samoré, 6 de junio de 2011. REUTERS/Iván Alvarado.

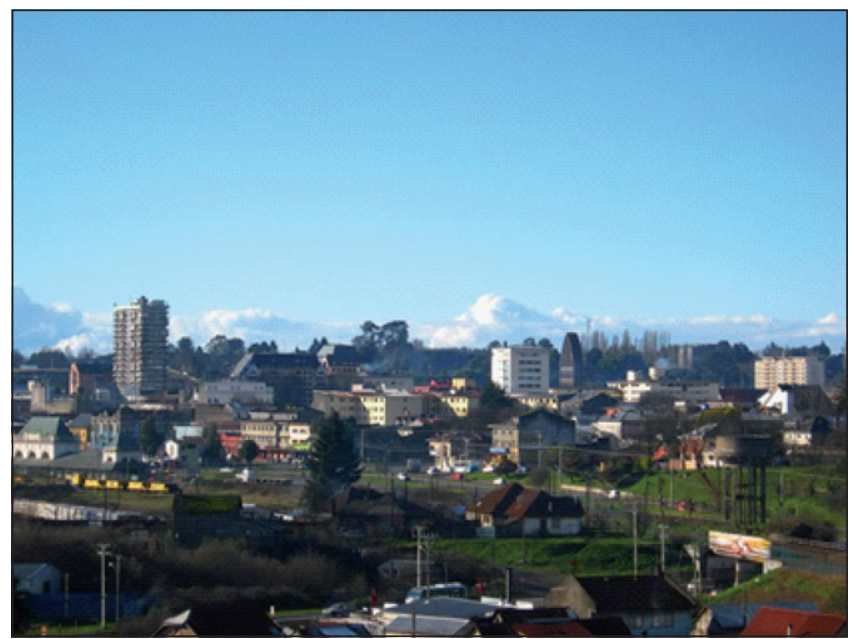

Figura 5. Fotografía panorámica de la ciudad de Osorno en verano.

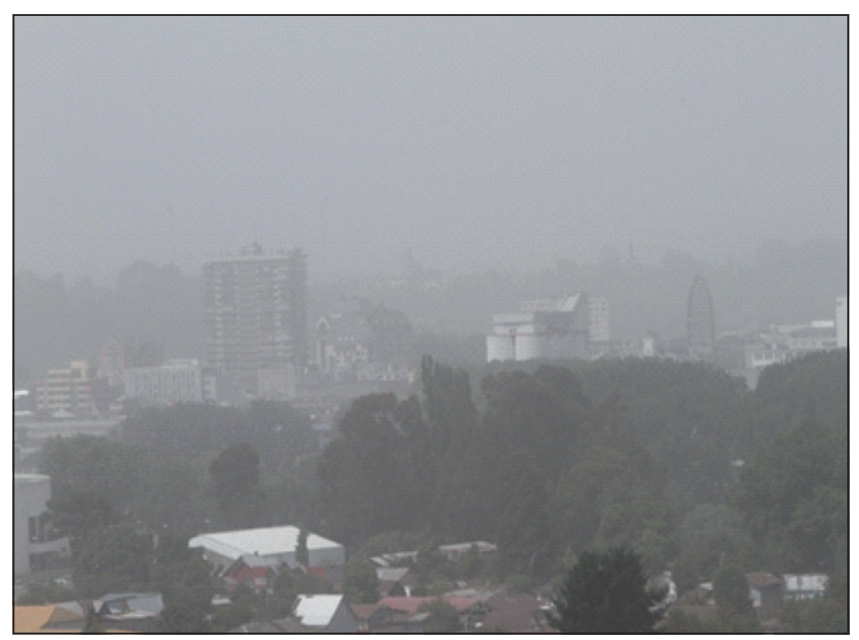

Figura 6. Ciudad de Osorno afectada por el esmog volcánico proveniente de las erupciones del cordón del Caulle (15:30 h del 12 de enero, 2012). del volcán (Figura 4). Su desplazamiento dependerá del viento. Conlleva un riesgo bajo de ocasionar daño a la salud, pero en personas portadoras de enfermedades respiratorias crónicas puede ser muy nociva. Además puede generar afecciones en la piel (dermatitis por irritación), oculares como abrasiones en la conjuntiva y en la córnea.

Los gases volcánicos se liberan antes, durante y después de la actividad volcánica, emanado al aire columnas de ceniza, polvo, dióxido de azufre, monóxido de carbono y otros gases dañinos. Estos productos químicos reaccionan con el oxígeno, el agua y la luz solar de la atmósfera para formar un tipo de contaminación del aire que se conoce como "esmog volcánico" (Figuras 5 y 6). Este esmog contiene una mezcla de gases y aerosoles de alto nivel de acidez, principalmente ácido sulfúrico, que en contacto con el agua genera "lluvia ácida". Estos aerosoles son lo suficientemente pequeños para ser inhalados y penetrar a las vías aéreas y espacios aéreos más profundos de los pulmones. Cuando las personas inhalan esta niebla volcánica, las membranas mucosas y las vías aéreas se irritan, y esto puede afectar la función pulmonar. Por ejemplo, la inhalación de $\mathrm{SO}_{2}$ produce broncoconstricción aguda observándose este efecto en sujetos asmáticos con concentraciones más bajas que en sujetos sanos ${ }^{2}$.

\section{Daños en la salud por cenizas y gases volcánicos}

Analizaremos por separado los efectos deletéreos sobre la salud de los diferentes componentes del "esmog volcánico".

Dióxido de carbono y ácido sulfhídrico: Los gases volcánicos más dañinos son aquellos que son más pesados que el aire (dióxido de carbono: $\mathrm{CO}_{2}$ y ácido sulfhídrico: $\mathrm{H}_{2} \mathrm{~S}$ ), pudiendo causar asfixia por desplazamiento del oxígeno en las zonas más bajas por acumulación. En 1986 en Camerún, una nube de $\mathrm{CO}_{2}$ causó la muerte de más de 1700 personas $^{3}$. Por su parte, los sulfuros producen asfixia y también intoxicación (inhibición de la citocromo oxidasa). La cefalea, la irritación ocular y respiratoria 
son signos precoces de su toxicidad, que en casos más graves puede producir edema pulmonar ${ }^{4}$. Se ha descrito que los sobrevivientes de estos episodios, generalmente presentan manifestaciones neuropsicológicas a largo plazo 5 .

Ácido fluorhídrico: A bajas concentraciones produce irritación de las vías aéreas superiores. En altas concentraciones puede generar úlceras de piel y mucosas ${ }^{6}$. También daño endotelial, broncoconstricción y edema pulmonar ${ }^{7}$. Además, se ha asociado a exacerbación de patologías respiratorias previas ${ }^{8,9}$. En agosto de 1991, la erupción del volcán Hudson, situado en Chile, en la Región de Aysén, depositó cenizas en la zona sur de Argentina contribuyendo a la muerte de miles de ovejas. El análisis de las cenizas detectó una elevada concentración de fluoruros, que ulteriormente se normalizó con las lluvias. Rubin y cols. estudiaron la presencia de fluorosis en personas y ovejas de la zona no encontrándose síntomas ni signos de ésta, ni tampoco aumento de la concentración de fluoruro en la orina de las ovejas por lo que se descartó esta entidad como la causa de la mortalidad ovina en esa erupción ${ }^{10}$.

Dióxido de azufre $\left(\mathrm{SO}_{2}\right)$ y silice $\left(\mathrm{SiO}_{2}\right)$ : Las partículas pequeñas que contienen $\mathrm{SO}_{2}$ (menor de $10 \mathrm{~mm}$ ) se asocian a irritación ocular, dolor faríngeo y exacerbación del asma especialmente por su efecto broncoconstrictor. Su exposición aumenta el riesgo de infecciones respiratorias y de bronquiolitis obliterante ${ }^{11}$. Pueden desplazarse hasta más de $30 \mathrm{~km}$ de distancia ${ }^{12}$.

La inhalación de sílice aumenta el riesgo de silicosis y ciertos minerales asbestiformes (zeo- lito y erionito) presentes en la ceniza se asocian a mesotelioma ${ }^{13}$.

Las erupciones volcánicas a través de sus emisiones de cenizas y efectos en el entorno pueden producir una amplia gama de condiciones patológicas en los habitantes de la zona amagada, pudiendo afectar especialmente a la piel y mucosas, al sistema respiratorio y al tracto gastrointestinal (Tabla 1).

\section{Mecanismos de daño pulmonar por inhalación de cenizas volcánicas}

Las cenizas volcánicas ocasionan patología respiratoria aguda en personas susceptibles, especialmente niños y en portadores de enfermedad pulmonar crónica ${ }^{14,15}$. La inflamación pulmonar comienza con el depósito de partículas en el pulmón. Su localización en el pulmón depende del patrón respiratorio determinado por el volumen corriente y la frecuencia respiratoria, la morfología de la vía aérea y el tamaño y forma de las partículas ${ }^{16}$.

En la vía aérea central las partículas son removidas por la tos y el barrido del sistema mucoci$\operatorname{liar}^{17}$. En las vías aéreas periféricas o bronquiolos (menor de $2 \mathrm{~mm}$ de diámetro y no ciliadas) la remoción de partículas la realizan los macrófagos alveolares y los capilares linfáticos ${ }^{18}$. Si las partículas son ultrafinas (menor de $0,1 \mathrm{~mm}$ ) pueden pasar directamente a la sangre que circula por los capilares pulmonares. Los macrófagos pueden liberar IL-1, IL-6, TNF (tumor necrosis factor),

Tabla 1. Condiciones patológicas producidas por las diferentes emisiones de las erupciones volcánicas

\begin{tabular}{|c|c|c|c|c|c|c|c|}
\hline $\begin{array}{l}\text { Condiciones } \\
\text { patológicas }\end{array}$ & Cenizas & Gases & $\begin{array}{l}\text { Flujo de } \\
\text { lava }\end{array}$ & Explosiones & $\begin{array}{l}\text { Nubes } \\
\text { ardientes }\end{array}$ & $\begin{array}{l}\text { Flujos de } \\
\text { lodo }\end{array}$ & $\begin{array}{c}\text { Lluvia } \\
\text { ácida }\end{array}$ \\
\hline $\begin{array}{l}\text { Infección respiratoria } \\
\text { Dermatitis, Irritación } \\
\text { ocular }\end{array}$ & $\checkmark$ & & & & & & \\
\hline $\begin{array}{l}\text { Enfermedades } \\
\text { gastrointestinales }\end{array}$ & $\checkmark$ & $\checkmark$ & $\checkmark$ & & & & $\checkmark$ \\
\hline Intoxicación & Fluorosis & $\begin{array}{l}\text { Por vía } \\
\text { aérea }\end{array}$ & $\begin{array}{l}\text { Por vía } \\
\text { aérea }\end{array}$ & & & & \\
\hline Quemaduras & & & $\begin{array}{l}\text { Piel y vías } \\
\text { respiratorias }\end{array}$ & $\begin{array}{l}\text { Piel y vías } \\
\text { respiratorias }\end{array}$ & $\begin{array}{l}\text { Piel y vías } \\
\text { respiratorias }\end{array}$ & Piel & \\
\hline Traumatismos & Secundarios & & $\checkmark$ & $\begin{array}{l}\text { Múltiples y } \\
\text { laceraciones }\end{array}$ & Múltiples & Múltiples & \\
\hline Sepsis & & & & & $\checkmark$ & $\begin{array}{c}\text { Sepsis y } \\
\text { amputaciones }\end{array}$ & \\
\hline $\begin{array}{l}\text { Trastornos } \\
\text { psicosociales }\end{array}$ & $\checkmark$ & $\checkmark$ & & & & & \\
\hline
\end{tabular}


FGF (fibroblast growth factor), y estimular la respuesta de los polimorfonucleares produciendo daño en el tejido pulmonar y amplificación de la respuesta inflamatoria. Esta cadena de reacciones se manifiesta como dificultad respiratoria y aumento en la susceptibilidad a las infecciones respiratorias. Más a largo plazo se puede producir fibrosis y carcinogénesis ${ }^{19}$.

La exposición breve a cenizas volcánicas altera las barreras defensivas físicas de las vías aéreas como la frecuencia del barrido ciliar y la barrera mucosa ${ }^{20}$. También se producen alteraciones en la inmunidad humoral. En este sentido se ha comunicado que trabajadores expuestos a cenizas volcánicas tienen una marcada disminución en los niveles séricos de $\mathrm{C} 3, \mathrm{C} 4$ e IgG, en comparación con trabajadores no expuestos ${ }^{21}$. También se ha señalado que IgA, IgG y albúmina protegen de los efectos nocivos de las partículas inhaladas ${ }^{22}$.

La exposición de animales de experimentación (roedores) a diferentes partículas volcánicas estimula a las células inmunocompetentes como los monocitos de manera más intensa que a los macrófagos alveolares en producir una respuesta oxidativa $^{23}$. La disminución en la producción de ión superóxido sugiere un riesgo aumentado de infección al comprometer la función fagocítica antibacteriana ${ }^{24}$. Los trabajos experimentales realizados con ceniza volcánica de la erupción de 1980 del volcán Santa Helena, EE.UU., han podido concluir que esta ceniza puede inducir fibrosis pulmonar y alterar funciones del macrófago in vitro. En nuestro país, Borzone y cols. evaluaron el efecto de las emanaciones de la erupción del volcán Lonquimay en 1989, exponiendo ratas Sprague-Dawley a respirar el aire de Malalcahuello, localidad cercana al volcán. Luego de 2,5 meses de exposición el estudio histopatológico detectó neumonitis intersticial en 10 de los 12 animales expuestos, no detectándose alteraciones del epitelio de la nariz, laringe, tráquea ni bronquios ${ }^{25}$.

\section{Síntomas respiratorios en la población expuesta}

Los síntomas respiratorios derivados de la inhalación de cenizas volcánicas dependen de múltiples factores: concentración de partículas en suspensión, proporción de partículas respirables (menor de $10 \mu \mathrm{m}$ de diámetro aerodinámico), duración y frecuencia de la exposición, presencia de sílice cristalina libre y de gases volcánicos o de aerosoles mezclados con la ceniza, condicio- nes meteorológicas, condiciones de la persona expuesta, v.gr. limitación ventilatoria obstructiva crónica (asma, EPOC, secuelas de TBC pulmonar) y del uso de equipos de protección respiratoria (máscaras). Según Blong, los síntomas respiratorios más frecuentemente señalados por las personas durante y después de una lluvia de cenizas volcánicas son: coriza; irritación y dolor faríngeo, acompañado a veces de tos seca; respiración inconfortable; las personas con afecciones pulmonares previas, desarrollaron síntomas respiratorios agudos intensos (tos, expectoración, sibilancias y disnea), que persistieron por algunos días después de la exposición. En general, estos efectos agudos no son considerados nocivos en personas sanas antes de la exposición. Sin embargo, son frecuentes las exacerbaciones de enfermedades respiratorias $(33 \%)$ y cardíacas pre-existentes después de la inhalación de ceniza volcánica ${ }^{26}$.

Las erupciones volcánicas afectan especialmente la salud respiratoria infantil como pudo comprobarse al analizar las consultas por problemas respiratorios en los servicios de urgencia luego de las erupciones del volcán Guagua Pichincha en Quito, Ecuador, en Abril del año 2000. Se constató aumento de las consultas por infecciones respiratorias agudas altas y bajas y descompensación de asma en la población pediátrica. Durante el período de expulsión de fumarolas el diagnóstico de asma se duplicó. Además, el mayor aumento de consultas por afecciones respiratorias se observó en los niños. Este estudio demostró la utilidad de los registros de los servicios de urgencia para monitorizar los efectos en la salud de la erupción volcánica. Distinguiéndose tres períodos en las consultas: 1) Síntomas relacionados con el efecto irritante agudo y transitorio de las cenizas volcánicas en la mucosa respiratoria alta y en los ojos; 2) Infecciones respiratorias de la vía aérea superior (IRA); 3) Infecciones respiratorias bajas, principalmente en pacientes con patología pulmonar crónica ${ }^{27}$.

Aunque existe limitada información sobre la mortalidad producida por las erupciones recientes, se conoce desde hace mucho tiempo que la mortalidad aguda de causa respiratoria en sujetos previamente sanos, se debería a la inhalación de gases tóxicos. Como fue el caso de Plinio $-E l$ Viejo-, naturalista romano, quien murió el año 79 d.C. al inhalar gases de azufre mientras investigaba la erupción del volcán Vesubio ${ }^{28}$.

Según una revisión de Gudmundsson, en relación a la erupción volcánica de 2011 en Eyjafjallajökull, Islandia, a corto plazo se registraron episodios de asma y bronquitis aguda en la po- 
blación expuesta. Si bien, no se han encontrado efectos a largo plazo de la exposición a ceniza volcánica sobre la función pulmonar, hay preocupación por el riesgo de silicosis luego de la exposición crónica a ésta ${ }^{29}$. Aunque aun no se ha demostrado que la exposición a las emanaciones de erupciones volcánicas produzca silicosis, hay que reconocer que en las últimas décadas sólo se han realizado estudios a largo plazo para evaluar el riesgo de silicosis luego de exposición crónica, en las erupciones del volcán Santa Helena (1980) y de Soufrière Hills (actualmente activo), en la isla Montserrat, Indias Británicas Occidentales. Aunque en rigor se necesitarían estudios de seguimiento de veinte años o más para evaluar los efectos respiratorios crónicos en la población expuesta, los estudios poblacionales realizados hasta ahora en ambas erupciones dieron evaluaciones diferentes de riesgo respiratorio a largo plazo. El estudio de seguimiento por cuatro años en leñadores después de la erupción del volcán Santa Helena indicó que no se necesitaba de más estudio, ya que las pruebas de función respiratoria fueron normales y las concentraciones de cenizas y el tiempo de exposición no alcanzaron la magnitud requerida para producir silicosis. En cambio, en el caso de la erupción de Soufrière Hills, es posible que algunos isleños hayan estado expuestos a una cantidad suficiente de ceniza volcánica para estar en riesgo de desarrollar silicosis, ya que la erupción se ha prolongado ya por 17 años, desde 1995 hasta ahora en que aún está activa y, por lo tanto, se requeriría de estudios de seguimiento a largo plazo para confirmar este riesgo.

Por lo tanto, en el caso de erupciones prolongadas por varios años, junto con monitorear la concentración y composición de las cenizas volcánicas y sus gases asociados, se debería efectuar un seguimiento a largo plazo de la población expuesta, no sólo por la posibilidad de desarrollar silicosis, sino que también por el riesgo de desarrollar EPOC y eventualmente cáncer pulmonar en relación con la inhalación de cristales de sílice u otros componentes de la ceniza volcánica como por ejemplo radón o titanio ${ }^{7}$.

En futuras erupciones volcánicas se ha recomendado instaurar un archivo de muestras de ceniza volcánica y un sitio web que contenga por una parte consejos para la población afectada $\mathrm{y}$ también información y una guía de procedimientos para los médicos y profesionales de la salud para enfrentar la emergencia y realizar estudios de evaluación de riesgos en la población expuesta con la participación de vulcanólogos y personal de los servicios de salud.
Considerando la situación producida por la erupción del Cordón del Caulle, el Ministerio de Salud del Gobierno de Chile, a través de la Secretaría Ministerial de Salud de Los Lagos, ha recomendado medidas de protección a la población expuesta, las cuales se resumen en la Tabla 2.

\section{Medidas de protección respiratoria ante erupciones volcánicas}

La estrategia básica es evitar la exposición innecesaria a la ceniza volcánica y usar una máscara facial apropiada cuando se está expuesto, especialmente en las operaciones de limpieza.

La evacuación de la población expuesta es una medida que muy rara vez se toma. Sin embargo, a las personas con enfermedades o síntomas respiratorios crónicos debería aconsejárseles que abandonen el área contaminada. Si las concentraciones respirables de sílice cristalina libre exceden el límite fijado por la NIOSH (National Institute for Occupational Safety Health de EE.UU.) de $50 \mathrm{mg} / \mathrm{m}^{3}$ por un período considerable de tiempo (por ejemplo meses o años) el riesgo de desarrollar silicosis debe ser considerado por las autoridades regionales y gubernamentales.

Lo aquí expuesto nos lleva a reflexionar que los hermosos volcanes de nuestro país pueden ser muy temperamentales en su comportamiento. Luego de cientos de años de estar formando parte del paisaje pacíficamente, pueden entrar en actividad súbitamente, provocando intranquilidad y alarma pública por los temblores y otros fenómenos que preceden a su erupción, la que al producirse puede generar una emergencia sanitaria de magnitud impredecible. Nuestra obligación inmediata, como profesionales de la salud y especialistas en enfermedades respiratorias, es contar con la mejor información para enfrentar racionalmente este problema de salud pública y las afecciones respiratorias que se generarán.

Concluimos señalando que el propósito central de esta revisión es entregar información sobre un problema de salud pública con el que cada cierto tiempo las fuerzas de la naturaleza nos golpean, ya que la última erupción volcánica ocurrida en nuestro país, puso en evidencia que necesitamos contar con mejor preparación e implementación de instrumentos que nos permitan enfrentar de la mejor manera esta emergencia.

\section{Agradecimientos}

Los autores agradecen el apoyo secretarial de la Sra. María Angélica Poblete de la Sociedad Chilena de Enfermedades Respiratorias. 
Tabla 2. Recomendaciones a la población en caso de erupción volcánica. Secretaría Ministerial Regional de Salud (SEREMI), X Región, Ministerio de Salud de Chile

\section{Protección ocular}

- Utilice gafas con protección lateral -no gafas de sol- cuando se encuentre en ambientes al aire libre. Puede usar los protectores que se recomiendan para ciclistas, motociclistas o nadadores.

- Si ha estado expuesto a la ceniza y presenta sensación de cuerpo extraño en sus ojos, lávelos con abundante agua. Es preferible utilizar agua potable y hervida. Lávese las manos antes del procedimiento. No utilice colirios o gotas oftalmológicas.

- Si la sensación de cuerpo extraño persiste luego del lavado es necesario solicitar atención médica.

- Las personas alérgicas tienen más probabilidad de desarrollar dermatitis en los párpados. En caso de presentar picazón excesiva, molestias con la luz o lagrimeo, deben consultar al oftalmólogo.

\section{Protección de la piel y el cuero cabelludo}

- Cúbrase con un sombrero, pañoleta o gorra para evitar el contacto de la ceniza con el cuero cabelludo.

- Utilice prendas que cubran la mayor extensión del cuerpo si va a exponerse al ambiente externo.

- Si la piel se ha impregnado de ceniza, quítese la ropa y lávese con abundante agua. Si presenta ardor o enrojecimiento de la piel, consulte con el médico.

\section{Protección del sistema respiratorio}

- En caso de abundante ceniza, la mejor protección es permanecer en ambientes cubiertos.

- Si debe movilizarse al exterior de su vivienda, use mascarillas comerciales o un pañuelo de tela húmedo para cubrir nariz y boca.

- En caso de abundante ceniza, las personas con enfermedades crónicas (asma), adultos mayores y niños deben permanecer en sus viviendas.

\section{Usar agua segura}

- Cubra las fuentes de agua como pozos y norias para que no les caiga ceniza.

- Para consumo prefiera agua embotellada o distribuida por camiones aljibes.

- Para higiene personal y de alcantarillado siga las instrucciones de la autoridad sanitaria.

\section{Prevención de accidentes}

- Si se ha recomendado la limpieza exterior de ceniza acumulada en techos, patios y calles, apoye las labores utilizando las medidas antes mencionadas para protegerse de la ceniza.

- La limpieza de los techos implica graves riesgos en caso de caídas. Infórmese y adopte todas las normas de seguridad y siga todas las recomendaciones para la limpieza y eliminación de la ceniza.

- Absténgase de conducir si la visibilidad está comprometida por la lluvia de ceniza.

- Si la precipitación de ceniza ha cesado y está acumulada en las calles, conduzca lentamente y dé prioridad a los vehículos oficiales, ambulancias y unidades de emergencia.

\section{Bibliografía}

1.- Volcanes en Chile. Sitio web:http://www.emol.com/ especiales/volcanes/chile.htm Consultado: 30.06.2012.

2.- OMS. Sector Salud. Frente al riesgo volcánico "Guía de preparativos de salud frente a erupciones volcánicas".

3.- WAGNER G, CLARK M, KOENIGSBERG E, DECATA S. Medical evaluation of the victims of the 1986 Lake Nyos disaster. J Forensic Sci 1988; 33:899-909.

4.- REIFFENSTEIN J, HULBERT W, ROTH S. Toxicology of hydrogen sulfide. Annu Rev Pharmacol Toxicol 1992; 32: 109-34.

5.- TVEDT B, SKYBERG K, AASERUD O, HOBBESLAND A, MATHIESEN T. Brain damage caused by hydrogen sulfide: a follow-up of six patients. Am J Ind Med 1991; 20: 91-101.

6.- SCIENTIFIC EVENT ALERT NETWORK (SEAN). Global volcanism 1975-1985: the first decade of reports from the Smithsonian Institution's Scientific Event Alert Network. Prentice-Hall. Nueva Jersey, 1989.

7.- WEISTEIN P, COOK A. Human health impacts of volcanic eruptions. Histopathology 2002; 41 (Suppl. 2): 329-33.

8.- OSTRO B, LIPSETT M, WIENER M, SELNER J. Asthmatic responses to airborne acid aerosols. Am J Public Health 1991; 81: 694-702.

9.- MANNINO D, RUBEN S, HOLSCHUH F, HOLSCHUH T, WILSON M. Emergency department visits and hospitalizations for respiratory disease on the Island of Hawaii, 1981 to 1991. Hawaii Med J 1996; 55 : 48-54.

10.- RUBIN C H, NOJI E K, SELIGMAN P J, HOLTZ J L, GRANDE J, VITTANI F. Evaluating a fluorosis hazard after a volcanic eruption. Arch Environ Health 1994; 49: 395-401.

11.- ELIAS T, SUTTON A J. Volcanic air pollution creates health concerns on the island of Hawaii. Cities on 
Volcanoes Conference Proceedings, Auckland, New Zealand, 2001.

12.- BAXTER P, STOIBER R, WILLIAMS S. Volcanic gases and health: Masaya Volcano, Nicaragua. Lancet 1982; 2: 150-1.

13.- ROHL A, LANGER A, MONCURE G, SELIKOFF I, FISCHBEIN A. Endemic pleural disease associated with exposure to mixed fibrous dust in Turkey. Science 1982; 216: 518-20.

14.- HORWELL C, BAXTER P. The respiratory health hazards of volcanic ash: a review for volcanic risk mitigation. Bull Volcanol 2006; 68: 1-24.

15.- BAXTER P. The eruption of El Reventador volcano 2002: health hazards and the implications for volcano risk management in Ecuador. Report to the Pan-American Health Organization, PanAmerican Health Organization, Washington DC, 2003.

16.- CULLEN R, JONES A, MILLER B, TRAN C, DAVIS J, DONALDSON K, et al. Toxicity of volcanic ash from Montserrat. Institute of Occupational Medicine 2002. (Research Report)

17.- LEHNERT B. Defense mechanisms against inhaled particles and associated particle-cell interactions (in health effects of mineral dusts). Rev Mineral 1993; 28: 425-69.

18.- MORROW P. Dust overloading of the lungs: update and appraisal. Toxicol Appl Pharmacol 1992; 113: 1-12.

19.- DRISCOLL K. Role of inflammation in the development of rat lung tumors in response to chronic particle exposure. Inhalat Toxicol 1996; 8: 139-53.

20.- SCHIFF L, BYRNE M, ELLIOTT S, MOORE S, KETELS K, GRAHAM J. Response of hamster trachea in organ culture to Mount St. Helens volcano ash. Scan Electron Microsc 1981; (Pt 2): 169-78.

21.- OLENCHOCK S, MULL J, MENTNECH N, LEWIS
D, BERNSTEIN R. Changes in humoral immunologic parameters after exposure to volcanic ash. J Toxicol Environ Health 1983; 11: 395-404.

22.- MARTIN T, WEHNER A, BUTLER J. Evaluation of physical health effects due to volcanic hazards: the use of experimental systems to estimate the pulmonary toxicity of volcanic ash. Am J Public Health 1986; 76 : $59-65$.

23.- BECKER S, SOUKUP J, GALLAGHER J. Differential particulate air pollution induced oxidant stress in human granulocytes, monocytes and alveolar macrophages. Toxicol In Vitro 2002; 16: 209-18.

24.- CASTRANOVA V, BOWMAN L, SHREVE J, JONES G, MILES P. Volcanic ash: toxicity to isolated lung cells. J Toxicol Environ Health 1982; 9: 317-25.

25.- BORZONE G, KLAASEN R, TORRES P, BORLANDO J. Efecto de la erupción del volcán Lonquimay sobre el aparato respiratorio de animales de laboratorio. Enferm Respir Cir Torác 1989; 5 (Supl): 17 (Resumen P 9).

26.- BLONG R. Volcanic hazards: a sourcebook on the effects of eruptions. Sydney: Academic Press Australia, 1984, págs. 83-91.

27.- NAUMOVA E N, YEPES H, GRIFFITHS J K, SEMPÉRTEGUI F, KHURANA G, JAGAI J S, et al. Emergency room visits for respiratory conditions in children increased after Guagua Pichincha volcanic eruptions in April 2000 in Quito, Ecuador observational study: time series analysis. Environ Health 2007; 6: 21-32.

28.- HAGGARD H. El médico en la Historia. Editorial Sudamericana, $2^{\mathrm{a}}$ edición, Buenos Aires, Argentina, 1943; págs. 105-8.

29.- GUDMUNDSSON G. Respiratory health effects of volcanic ash with special reference to Iceland. A review. Clin Respir J 2011; 5: 2-9.
Correspondencia a:

Dr. Edgardo Grob B.

Campo Clínico Osorno.

Facultad de Medicina,

Universidad Austral de Chile.

Osorno XIV Región.

E-mail: edgrob1@hotmail.com 